\title{
Erratum: Microscopic description of exciton-polaritons in microcavities [Phys. Rev. Research 1, 033120 (2019)]
}

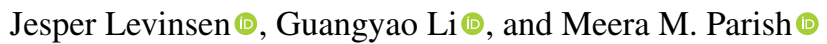 \\ (Received 3 September 2021; published 16 September 2021)
}

DOI: 10.1103/PhysRevResearch.3.039003

In our original article, there was an issue with the numerical evaluation of the real-space electron-hole wave function $\tilde{\varphi}(r)$ for the upper polariton. This mistake affected Figs. 4(a) and 4(b) and we show the corrected figure here. As we can see from Figs. 4(a) and 4(b), when the energy of the upper polariton approaches the $2 s$ exciton state at large Rabi coupling, its electron-hole wave function inherits a node from the $2 s$ exciton wave function, which was absent in the original figure. Figure 4(e) remains visually unchanged because the average value is given by the full spatial extension of the wave function instead of merely the short-range feature shown in Figs. 4(a) and 4(b). This issue did not affect the lower polariton; however, for completeness we show the corresponding plots in the bottom row of the figure. This correction does not affect any of the main results of the article.

We thank Emma Laird for alerting us to this problem and for double checking our numerics.
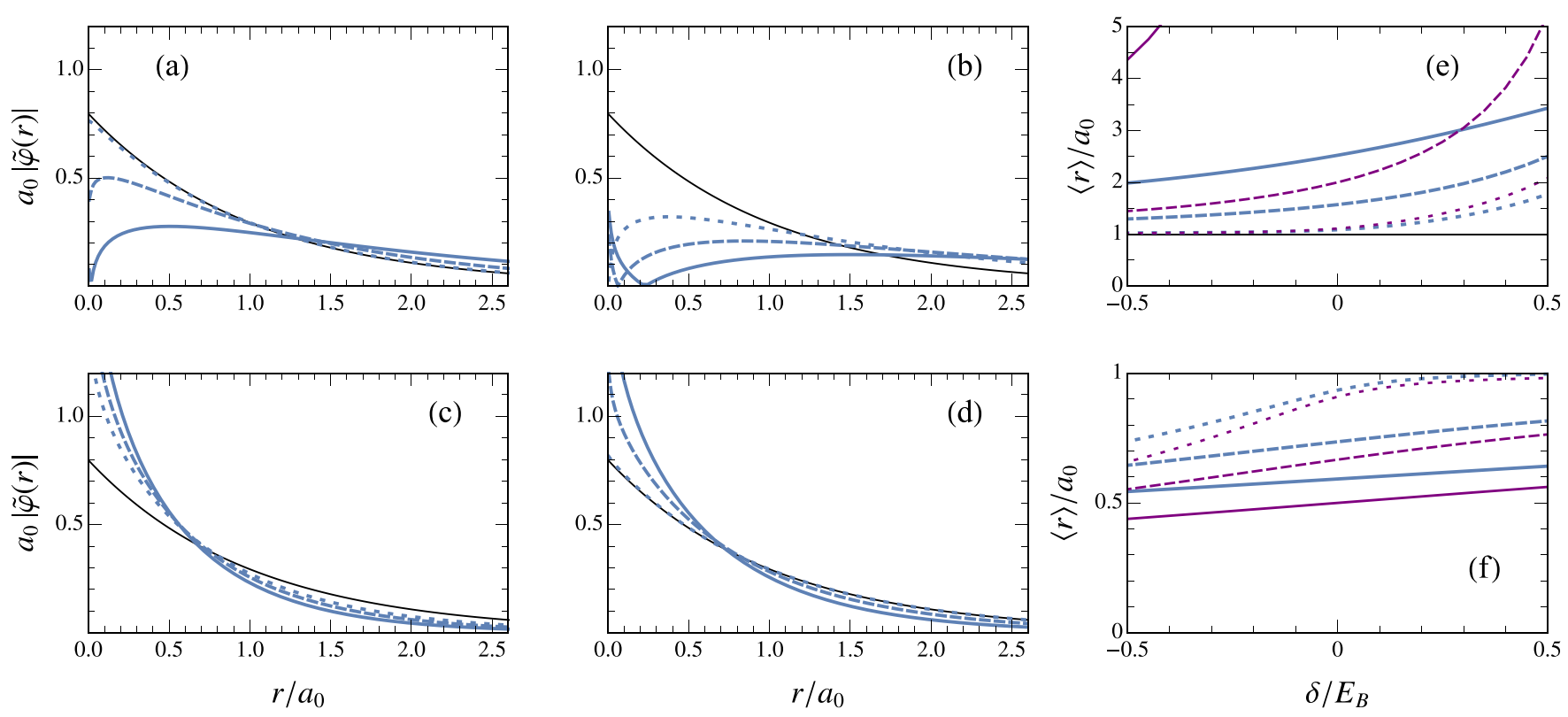

FIG. 4. (a)-(d) Normalized electron-hole wave function as a function of separation, and (e), (f) the mean electron-hole separation as a function of detuning. The upper (lower) panels correspond to the upper (lower) polariton, and we take $\delta / E_{\mathrm{B}}=-0.5$ in (a) and (c) and $\delta / E_{\mathrm{B}}=0.5$ in (b) and (d). We show the exact results obtained from our microscopic theory (blue lines) for the Rabi coupling strengths of $\Omega / E_{\mathrm{B}}=0.1$ (dotted line), $\Omega / E_{\mathrm{B}}=0.5$ (dashed line), and $\Omega / E_{\mathrm{B}}=1$ (solid line). Note that the numerically calculated wave functions in (a)-(d) are only shown for $r>0.005 a_{0}$. We also show the $1 s$ exciton wave function and average separation (thin black line). In (e) and (f), the purple lines correspond to the result (27) from the variational approach [1,2], where we take the detuning to be $\tilde{\delta}$ (see main text).

[1] J. Khurgin, Excitonic radius in the cavity polariton in the regime of very strong coupling, Solid State Commun. 117, 307 (2001).

[2] H. Zhang, N. Y. Kim, Y. Yamamoto, and N. Na, Very strong coupling in GaAs-based optical microcavities, Phys. Rev. B 87, 115303 (2013).

Published by the American Physical Society under the terms of the Creative Commons Attribution 4.0 International license. Further distribution of this work must maintain attribution to the author(s) and the published article's title, journal citation, and DOI. 